\title{
Projeto de um calorímetro de relaxação para ensino de Física+*
}

Fábio Saraiva da Rochal

Universidade Federal de Pelotas

Pelotas - RS

Paulo Henrique Guadagnini ${ }^{2}$

Marcia Maria Lucchese ${ }^{3}$

Unipampa Bagé

Bagé - RS

\section{Resumo}

Apresentamos o projeto de um calorímetro de relaxação para medidas de calor específico em temperatura ambiente direcionado ao ensino de física experimental. Apontamos as características de constituição física e de operação do calorímetro e detalhamos a programação computacional envolvida. No transcorrer da experiência, o monitoramento da temperatura e do tempo foi feito eletronicamente em tempo real por um computador conectado a uma placa Arduino. Os dados obtidos podem ser disponibilizados por tabelas e gráficos através da ferramenta de aquisição de dados PLX-DAQ e a planilha Excel. Os resultados do calor específico médio obtidos para amostras de ferro, latão e chumbo, em temperatura ambiente, apresentam pequenos desvios da ordem de aproximadamente $3 \%$ quando comparados a valores da literatura. A proposta apresentada utiliza materiais de baixo custo e alta tecnologia objetivando a inovação dos laboratórios didáticos para ensino de ciências ou o desenvolvimento de atividades experimentais em componentes curriculares de instrumentação para ensino de Física.

\footnotetext{
${ }^{+}$Relaxation calorimeter design for Physics teaching

* Recebido: junho de 2016.

Aceito: fevereiro de 2017.

${ }^{1}$ E-mail: fabio.saraiva.rocha@gmail.com

2 E-mail: pguadagnini@gmail.com

3 E-mail: mmlucchese@gmail.com
} 
Palavras-chave: Ensino de Física; Calorímetro de Relaxação; Arduino.

\begin{abstract}
In this work, we present the design of a relaxation calorimeter for specific heat measurements at room temperature directed to experimental Physics teaching. We point out the characteristics of physical constitution and calorimeter operation and detail the computer programming involved. In the course of the experiment, monitoring the temperature and time of the specific heat measurements were made electronically in real time by a computer connected to an Arduino board. The data can be made available through tables and graphs through PLX-DAQ data acquisition tool and Excel spreadsheet. The results of the average specific heat obtained for iron, brass and lead samples, at room temperature, have small deviations in the order of about $3 \%$ when compared to literature values. The proposal uses low-cost and high-tech equipment aiming at innovation of teaching laboratories for science education or the development of experimental activities in instrumentation disciplines for teaching Physics.
\end{abstract}

Keywords: Physics Teaching; Relaxation Calorimeter; Arduino.

\title{
I. Introdução
}

As discussões sobre atividades experimentais no ensino de Física são inúmeras, tanto no sentido da utilização de material demonstrativo em sala de aula convencional quanto no sentido de proporcionar atividades abertas nas quais os alunos podem pensar, criar e refletir sobre o que estão executando (BORGES, 2002). A atividade experimental não somente contribui para o aprendizado teórico quanto proporciona habilidades de manipulação de experimentos com trabalhos em grupos, de organização e questionamento de resultados e suas validades na forma de tabelas e gráficos, de investigação de possíveis problemas inerentes às medidas (SÉRÉ; COELHO; NUNES, 2003). Silva et al. (2005) apresentam em seu trabalho, que a aquisição dos dados via computador em experimentos de física deixa os alunos mais motivados em comparação com as atividades similares do laboratório tradicional.

Atualmente, as práticas de laboratório didático de Física nas instituições de ensino, geralmente, vêm com roteiros prontos (engessados) e muitas vezes mal elaborados, construídos com materiais inadequados e que acabam desestimulando o aluno na hora de realizar e interpretar o experimento. Tais práticas pouco ajudam os alunos a perceberem o vínculo da experimentação com teorias e leis físicas subjacentes. Um exemplo notório são as atividades de calorimetria. 
O calorímetro de relaxação apresentado neste trabalho poderá ser utilizado como base para o desenvolvimento de projetos e atividades experimentais abertas nas quais a própria construção ou modificação do equipamento pode ser explorada pelos estudantes.

Tradicionalmente os calorímetros didáticos disponíveis nas escolas de ensino médio e nas universidades são do tipo adiabáticos e vendidos em conjuntos de materiais instrucionais, "kit". Via de regra, são feitos de garrafas térmicas de uso comercial e não possuem bom isolamento térmico durante a execução da prática. Normalmente, o objetivo é unicamente medir o calor específico de uma determinada substância metálica e compará-lo ao valor tabelado e disponível no livro texto (AXT; GUIMARÃES, 1991). Nestes experimentos as diferenças entre os valores experimentais obtidos pelos alunos e os valores teóricos são grandes devido as trocas espúrias de calor que acontecem durante o experimento e devido a imprecisão nas medidas de temperatura feitas através de termômetro tradicional de bulbo e coluna de mercúrio. Apesar de ser possível obter bom nível de exatidão em experimentos utilizando calorímetros adiabáticos que sejam cuidadosamente calibrados e operados, a realidade no laboratório didático é que os estudantes usualmente não obtêm resultados de exatidão satisfatória utilizando calorímetros adiabáticos simples.

O projeto detalhado neste trabalho é de inovação tecnológica e tem por finalidade apresentar a construção de um protótipo de calorímetro de relaxação de baixo custo para medidas automatizadas de calor específico de corpos sólidos na temperatura ambiente através do uso de uma fonte de energia de computador encontrada em sucata, termômetros de estado sólido, uma plataforma microcontrolada (Arduino) e um computador. Com projetos deste tipo desejamos promover maior acessibilidade às tecnologias disponíveis atualmente e suprir parte da demanda de renovação do instrumental de coleta de dados de laboratórios didáticos que não podem contar com grandes recursos financeiros para a compra de equipamentos comerciais modernos.

Apresentamos as características físicas básicas do calorímetro e de todo o processo de medida, além de discutir os resultados alcançados e suas validades. O experimento que propomos pode ser reproduzido com baixo investimento financeiro em um laboratório de ensino de Física, como projeto numa classe de instrumentação para ensino de Física ou até mesmo em uma sala de aula para fins demonstrativos.

\section{Calor Específico}

A quantidade de energia na forma de calor necessária para alterar a temperatura de uma substância é proporcional a variação de temperatura $(\Delta T)$ e a capacidade térmica (ou calorífica) da substância $(C)$, veja a Equação 1, (YOUNG; FREEDMAN, 2003).

$$
Q=C \cdot \Delta T
$$


A capacidade térmica por unidade de massa $(m)$ de um material específico é denominada de capacidade térmica específica. O calor específico (c) é característico do material do qual o corpo é constituído, veja a Equação 2, (YOUNG; FREEDMAN, 2003).

$$
C=\frac{c}{m}=\frac{Q}{m \cdot \Delta T}
$$

Sendo assim o calor específico pode ser quantificado como a energia, em calorias, necessária para elevar em $1^{\circ} \mathrm{C}$ a temperatura de $1 \mathrm{~g}$ de determinada substância, ou no Sistema Internacional de unidades (SI), é a quantidade de energia, em Joules, necessária para aumentar em $1 \mathrm{~K}$ a temperatura de $1 \mathrm{~kg}$ da substância. Nem a capacidade térmica de um corpo nem o calor específico de um material são constantes, ambos dependem da temperatura e também da pressão. As equações (1) e (2) fornecem valores médios para estas grandezas na faixa de temperatura $\Delta T$. Por exemplo, nos livros texto normalmente consta o calor específico da água como sendo $1 \mathrm{cal} / \mathrm{g} .{ }^{\circ} \mathrm{C}(4187 \mathrm{~J} /(\mathrm{kg} \mathrm{K})$. Este valor varia menos de $1 \%$ no intervalo que vai de 0 até $100^{\circ} \mathrm{C}$.

Para determinação do calor específico é necessário manter ou pressão constante, denominado $\left(c_{p}\right)$ ou volume constante $\left(c_{v}\right)$. Em sólidos e líquidos os valores para $c_{p}$ e $c_{v}$ não variam muito, já em gases a variação é significativa. Neste trabalho, as medidas de calor específico $\left(c_{p}\right)$ mostradas foram feitas em temperatura ambiente e pressão atmosférica aproximadamente constante.

\section{Técnica de medida de capacidade calorífica utilizando um calorímetro de relaxação}

Um esquema simplificado do calorímetro de relaxação proposto neste trabalho é apresentado na Fig. 1. A amostra é posicionada sobre um porta-amostra no qual é fixado um aquecedor elétrico e um sensor de temperatura. A conexão térmica entre a amostra e o portaamostra é feita através de uma pasta térmica de alta condutividade térmica. É utilizada uma conexão térmica permanente entre o porta-amostra e um reservatório térmico de baixa temperatura (reservatório frio), o qual é mantido em uma temperatura constante. Os componentes do calorímetro são mantidos no interior de um recipiente com paredes de baixa condutividade térmica de modo a minimizar as trocas térmicas entre os componentes do calorímetro e o ambiente externo (adiabaticidade).

A operação do calorímetro de relaxação consiste em aplicar um pulso de energia térmica ligando-se o aquecedor e desligando-o após um tempo determinado (ciclo de aquecimento), e em seguida medindo-se o perfil de decaimento da temperatura, medida com o sensor de temperatura (ciclo de relaxação). A Fig. 2 mostra o comportamento térmico do sensor de temperatura em contato com o porta-amostra durante os ciclos de aquecimento e relaxação, e a variação de potência do aquecedor durante o ciclo de aquecimento. 


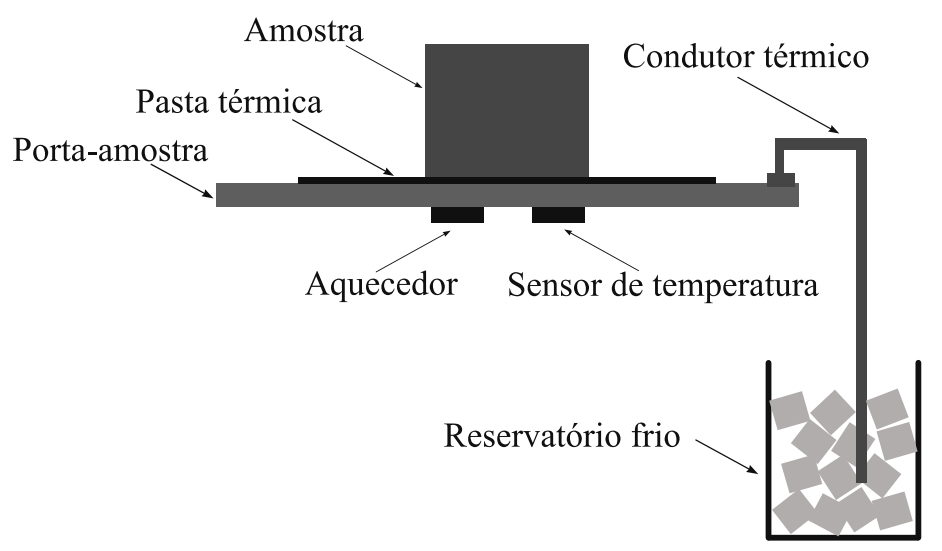

Fig. 1 - Esquema de construção do calorímetro de relaxação indicando as conexões térmicas entre os seus componentes.

Antes de se iniciar o ciclo de aquecimento, a amostra e o porta-amostra encontram-se na temperatura do reservatório frio, $T_{0}$. No ciclo de aquecimento, um pulso de energia térmica de potência $P_{0}$ e duração $t_{1}-t_{0}$ é aplicado no porta-amostra. A duração do pulso de energia térmica é especificada de modo que o sistema atinja o estado estacionário para condução de energia térmica entre o aquecedor, o porta-amostra e o reservatório térmico. Este estado é caracterizado pela estabilidade da temperatura do conjunto amostra mais porta-amostra, que atinge o seu valor máximo, $T_{\max }$. Após o desligamento do aquecedor, inicia-se o ciclo de relaxação em que o sistema, consistindo da amostra e do porta-amostra, transfere energia térmica para o reservatório frio, reduzindo a temperatura do porta-amostra até $T_{0}$.

A Fig. 3 representa o modelo para o fluxo unidimensional de energia térmica entre a amostra, o porta-amostra e o reservatório frio. Neste modelo, o reservatório frio é caracterizado por uma temperatura constante, $T_{0}$, e é conectado ao porta-amostra por um meio condutor de energia térmica de condutância térmica $k$. O porta-amostra, cuja temperatura variável é $T_{p}(t)$, é caracterizado por uma capacidade calorífica $C_{p}$. A amostra, cuja capacidade calorífica é $C_{a}$, é conectada termicamente ao porta-amostra através de um meio de condutância térmica $k^{\prime}$. Devido ao uso de uma pasta de elevada condutividade térmica para conexão entre a amostra e o porta amostra, assume-se um contato térmico ideal entre estes dois componentes, e considerase a condutância térmica $k^{\prime} \rightarrow \infty$. Segundo esta aproximação, as temperaturas da amostra, $T_{a}(t)$, e do porta-amostra, $T_{p}(t)$, serão iguais em qualquer instante do tempo: $T_{a}(t)=T_{p}(t)=$ $T_{s}(t)$, em que $T_{s}(t)$ é a temperatura do sistema amostra mais porta-amostra, e tal sistema será caracterizado pela capacidade térmica $C_{s}$.

Para modelar o fluxo unidimensional de energia térmica entre os componentes do calorímetro de relaxação utiliza-se a Equação 3 (ANDERSON, 2013).

$$
P(t)=C_{s} \frac{d T(t)}{d t}+k\left[T_{s}(t)-T_{0}\right]
$$




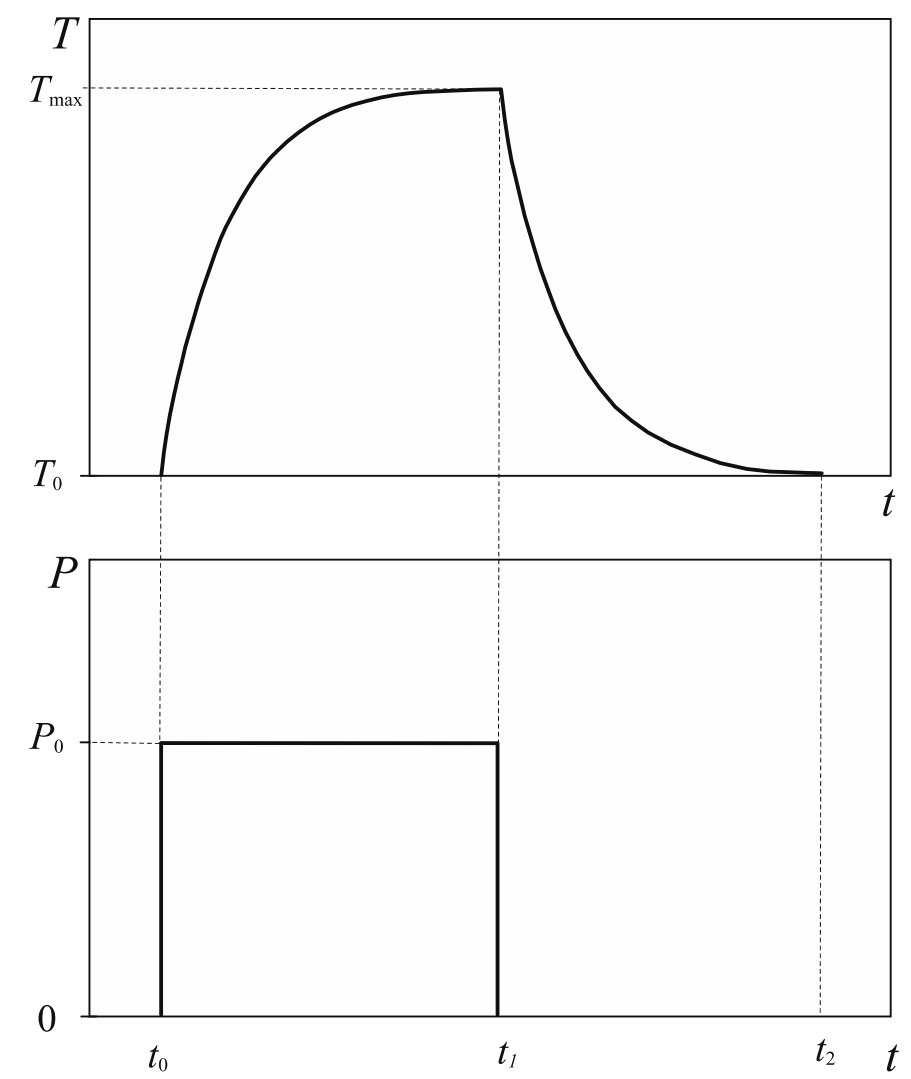

Fig. 2 - Comportamento térmico do termômetro acoplado ao porta-amostra durante os ciclos de aquecimento e relaxação, e a variação de potência do aquecedor durante o ciclo de aquecimento

Durante o ciclo de aquecimento, energia térmica é transferida para o sistema amostra mais porta-amostra na taxa $P(t)$. Parte desta energia é acumulada no sistema amostra mais porta-amostra (na taxa descrita pelo primeiro termo à direita do sinal de igualdade na Equação 3) e parte é transferida para o reservatório frio (na taxa descrita pelo segundo termo à direta do sinal de igualdade na Equação 3). Deve-se observar que, à medida que energia é transferida para o sistema amostra mais porta-amostra, a temperatura $T(t)$ aumenta, elevando a taxa de transferência de energia para o reservatório frio. Aguardando-se um tempo suficientemente longo, o sistema entra em estado estacionário (também chamado de estado permanente), no qual a taxa de entrada de energia térmica no sistema amostra mais porta-amostra (gerada pelo aquecedor) iguala-se à taxa de transferência de energia térmica do sistema amostra mais portaamostra para o reservatório frio.

$$
\Delta T_{\max , s}=T_{\max , s}-T_{0}
$$

No estado estacionário, a temperatura do sistema amostra mais porta-amostra mantém- 
se constante e igual ao seu valor máximo $T_{\max , s}$, resultando em um valor máximo para a diferença de temperatura entre o sistema amostra mais porta-amostra e o reservatório frio, $\Delta T_{\max , s}($ Equação 4).

A partir das Equações 3 e 4, e considerando-se que a potência fornecida pelo aquecedor é constante e igual a $P_{0}$, pode-se deduzir que no estado estacionário:

$$
P_{0}=k \Delta T_{\max , s}
$$

Após o desligamento do aquecedor $\left(P_{0}=0\right)$, inicia-se o ciclo de relaxação com a condução de parte da energia térmica armazenada no sistema amostra mais porta-amostra para o reservatório frio através da conexão térmica destes dois componentes. A partir da Equação 3, a equação para o fluxo unidimensional de energia térmica para o ciclo de relaxação torna-se a Equação 6.

$$
C_{S} \frac{d T(t)}{d t}+k\left[T_{S}(t)-T_{0}\right]=0
$$

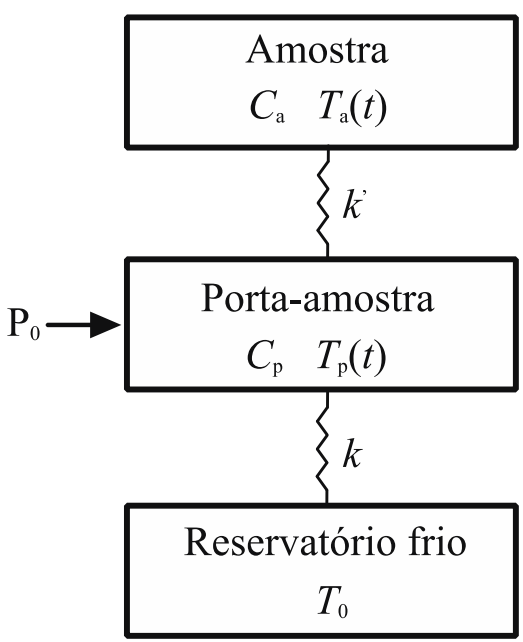

Fig. 3 - Modelo de fluxo unidimensional de energia térmica envolvendo a amostra, porta-amostra e reservatório frio.

Resolvendo-se a Equação 6 com a condição de contorno de $T(t)=T_{\max , s}$ quando $t=0$ (momento em que o aquecedor é desligado), obtém-se a Equação 7, que sugere um decaimento exponencial da temperatura do sistema amostra mais porta-amostra durante o ciclo de relaxação até que esta atinja o valor de $T_{0}$.

$$
\left[T(t)-T_{0}\right]=\Delta T_{\max , s} e^{\frac{-k}{C_{s}} t}
$$

O tempo de relaxação, $\tau_{S}$, é definido seguindo a Equação 8 e pode ser interpretado como o tempo, contado a partir do desligamento do aquecedor, no qual a diferença de temperatura entre o sistema amostra mais porta-amostra e o reservatório frio cai a cerca de $37 \%$ de seu valor máximo $\left(\Delta T_{\max , s}\right)$. 


$$
\tau_{s}=\frac{C_{s}}{k}
$$

A Equação 7 pode ser reescrita utilizando a definição do tempo de relaxação, resultando na Equação 9.

$$
\left[T(t)-T_{0}\right]=\Delta T_{\max , s} e^{\frac{-t}{\tau_{s}}}
$$

Os dados experimentais de temperatura coletados em função do tempo durante o ciclo de relaxação podem ser utilizados para efetuar um ajuste de curva para a função exponencial representada na Equação 9. Como resultado deste ajuste de curva, obtém-se $\Delta T_{\max , s} \mathrm{e} \tau_{s}$.

Conhecendo-se a potência do aquecedor, $P_{0}$, e o valor estimado experimentalmente de $\Delta T_{\max , s}$, pode-se utilizar a Equação 5 para obter-se a condutância térmica efetiva entre o sistema amostra mais porta-amostra e o reservatório frio (Equação 10).

$$
k=\frac{P_{0}}{\Delta T_{\max , s}}
$$

Combinando-se as Equações 8 e 10 pode-se obter a capacidade calorífica do sistema amostra/porta amostra, segundo a Equação 11.

$$
C_{s}=\frac{\tau_{s} P_{0}}{\Delta T_{\max , s}}
$$

Repetindo o experimento com os ciclos de aquecimento e relaxação sem que a amostra esteja posicionada no porta-amostra, pode-se obter a capacidade calorífica do porta amostra $\left(C_{p}\right)$ e o tempo de relaxação do porta-amostra $\left(\tau_{p}\right)$. Ambos valores são inferiores aos determinados para o sistema amostra mais porta-amostra. A capacidade calorífica do porta-amostra poderá ser determinada segundo o procedimento descrito acima utilizando a Equação 12, em que $\Delta T_{\max , p}$ é a variação de temperatura máxima determinada para o experimento em que a amostra não se encontra presente no porta-amostra.

$$
C_{p}=\frac{\tau_{p} P_{0}}{\Delta T_{\max , p}}
$$

A capacidade calorífica da amostra, $C_{a}$, pode ser calculada pela diferença entre a capacidade calorífica do sistema amostra mais porta-amostra e a capacidade calorífica do portaamostra, segundo a Equação 13.

$$
C_{a}=C_{s}-C_{p}
$$

Os valores de $C_{p}$ e $C_{S}$ são obtidos, respectivamente, utilizando as Equações 11 e 12. Para tal, são coletados dados de temperatura em função do tempo do porta-amostra (vazio) e do sistema amostra mais porta-amostra de modo a obter-se os tempos de relaxação e variações máximas de temperatura.

O calor específico da amostra, $c_{a}$, é calculada segundo a Equação 14 , em que $m_{a}$ é a massa da amostra. 


$$
c_{a}=\frac{C_{a}}{m_{a}}
$$

Para obter-se o calor específico do material constituinte da amostra, é necessário conhecer-se a potência do aquecedor, $P_{0}$. Para tal, é realizado um procedimento de calibração em que é utilizada uma amostra padrão (usualmente de alumínio) cuja capacidade calorífica é conhecida, $C_{A l}$. A potência do aquecedor pode ser calculada combinando as Equações 11, $12 \mathrm{e}$ 13 considerando $C_{a}=C_{A l}$, segundo a Equação 15 (STEIMACHER, 2008).

$$
P_{0}=C_{A l}\left[\frac{\tau_{s}}{\Delta T_{\max , s}}-\frac{\tau_{p}}{\Delta T_{\max , p}}\right]^{-1}
$$

\section{O projeto do calorímetro de relaxação para medidas em temperatura ambiente}

O calorímetro proposto é constituído de uma caixa de poliestireno expandido (isopor) de dimensões aproximadas de $15 \mathrm{~cm}$ x $18 \mathrm{~cm}$ x $55 \mathrm{~cm}$ (encontrada em mercados comuns) com paredes de espessura aproximada de $2 \mathrm{~cm}$ que serve como receptáculo para o porta-amostra (lâmina de alumínio de dimensões aproximadas de 4,5 cm x 6,0 cm, espessura de 1,00 mm e massa de 3,7 $\pm 0,1 \mathrm{~g}$ ) com um termômetro e um aquecedor acoplados. Também se encontra dentro da caixa de isopor um cilindro de alumínio aberto em uma de suas extremidades (no formato de um copo) com raio da base $4 \mathrm{~cm}$, altura $10 \mathrm{~cm}$ e paredes de espessura de 1,0 mm.

Do porta-amostra, através de um terminal anel fixado com parafuso, sai uma malha de cobre de comprimento aproximado de $16 \mathrm{~cm}$, largura 3,0 mm e espessura de 1,0 $\mathrm{mm}$ (marca Hikari modelo HK 120-05) fazendo o contato térmico com a blindagem através de um pino banana PB21. A blindagem é resfriada por contato térmico com um reservatório de alumínio de dimensões $5,5 \mathrm{~cm}$ x $14,0 \mathrm{~cm}$ x 23,0 cm que permanece com seu volume de cerca de $1770 \mathrm{~cm}^{3}$ preenchidos com gelo durante toda a experiência para garantir a estabilidade da temperatura da reservatório frio próximo de $0{ }^{\circ} \mathrm{C}$. O reservatório de gelo é recoberto com outra caixa de isopor com dimensões aproximadas de $18,0 \mathrm{~cm}$ x 22,0 cm x 28,0 cm para dificultar as trocas térmicas com o ambiente externo e garantindo uma maior estabilidade da temperatura da blindagem por longo tempo.

O calorímetro possui suporte de equipamentos eletrônicos para seu funcionamento. O aquecedor utilizado no calorímetro é baseado na dissipação de calor do terminal coletor de um transistor modelo TIP 41C que é acoplado ao porta-amostra através de uma lâmina de mica e pasta térmica, veja Fig. 4. O transistor é alimentado pela saída de $12 \mathrm{~V}$ de uma fonte ATX obtida de sucata de computador (esta fonte pode também ser adquirida no mercado de sucata de informática por um baixo custo monetário), veja Fig. 4. Devido à natureza da fonte, é necessário que a saída de $5 \mathrm{~V}$ receba uma carga durante as medidas com o intuito de estabilizar as 
tensões de saída. A carga escolhida foi um resistor de $5 \Omega$ com $10 \mathrm{~W}$ de dissipação. O esquema elétrico do circuito simples de alimentação do aquecedor também pode ser visto na Fig. 4.

Neste circuito a resistência colocada entre a alimentação de $12 \mathrm{~V}$ da fonte e a base do TIP é de $6,30 \mathrm{k} \Omega$ (obtidos através de um potenciômetro linear de $10 \mathrm{k} \Omega$ ). Um amperímetro colocado em série com a saída de $12 \mathrm{~V}$ da fonte apresenta valores típicos de corrente drenada entre 0,33 A e 0,47 A durante uma medida que normalmente apresenta valores de $3{ }^{\circ} \mathrm{C}$ ou $4{ }^{\circ} \mathrm{C}$ de estabilidade na temperatura do sistema até valores que podem chegar a cerca de $74{ }^{\circ} \mathrm{C}$ no caso da medição da capacidade térmica apenas relacionada ao porta-amostra.

O monitoramento da tensão de saída da fonte apresentou variação máxima de $2 \%$ durante a medida. A variação ocorrida na corrente entregue pela fonte decorre principalmente da alteração na resistência elétrica do circuito interno do transistor devido a variação de temperatura. $\mathrm{O}$ circuito de medição da temperatura do porta-amostra é composto de um termômetro de estado sólido de precisão tipo LM35, veja Fig. 4, que pode ser adquirido no mercado de componentes eletrônicos por um baixo custo monetário.

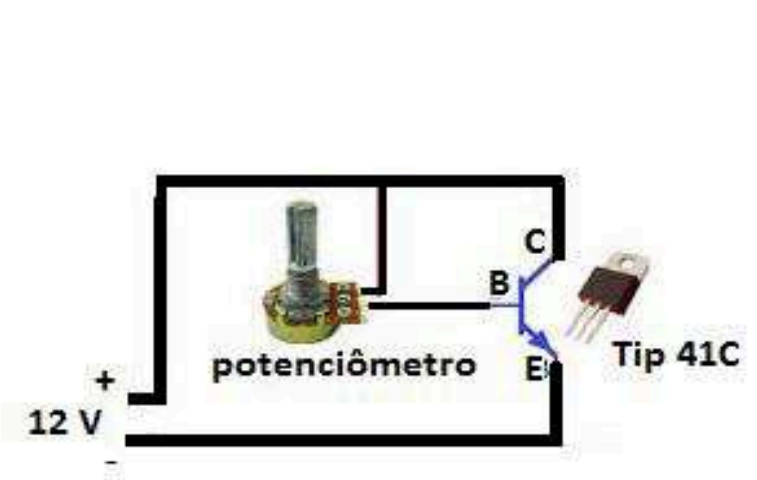

(a)

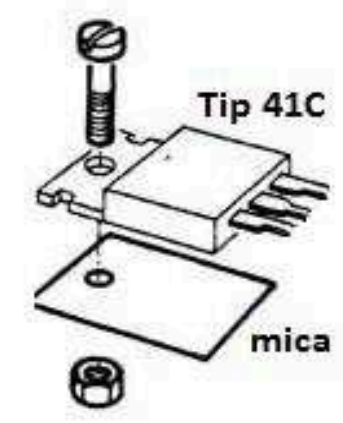

(b)

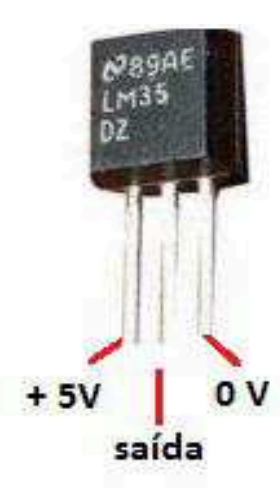

(c)

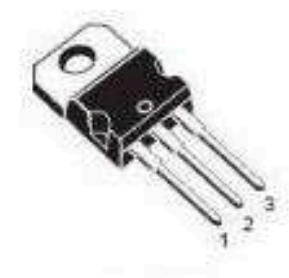

Tip 41C

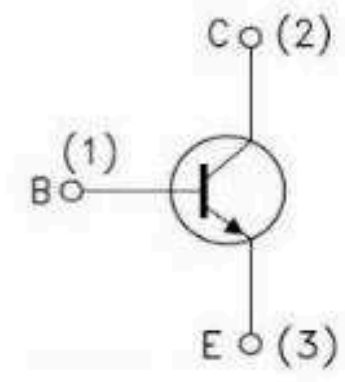

(d)

Fig. 4 - Em (a) o esquema elétrico de alimentação do aquecedor do porta-amostra, em (b) seu esquema de fixação mecânica no porta-amostra, em (c) a indicação de alimentação do LM35 e em (d) as indicações de pinagem para alimentação do transistor TIP 41C. 
O sensor LM35 apresenta uma saída de tensão linear relativa à temperatura em que ele se encontrar no momento em que for alimentado por uma tensão contínua de $4 \mathrm{~V}$ até $20 \mathrm{~V}$. Sua saída apresenta tensão de $10 \mathrm{mV} /{ }^{\circ} \mathrm{C}$. O sensor não necessita de qualquer calibração externa fornecendo com exatidão valores de temperatura com variações entre $0,25^{\circ} \mathrm{C}$ e $0,75^{\circ} \mathrm{C}$ dentro da faixa de temperatura de $-55^{\circ} \mathrm{C}$ a $150{ }^{\circ} \mathrm{C}$. Outro aspecto importante é que este sensor drena apenas $60 \mu \mathrm{A}$ durante seu funcionamento, sendo seu autoaquecimento de aproximadamente 0,1 ${ }^{\circ} \mathrm{C}$ ao ar livre. O sensor LM35 tem o encapsulamento mais comum TO-92 se parecendo com um transistor e facilitando o acoplamento com o porta-amostra apenas através de pasta térmica comum encontrada no comércio de componentes eletrônicos por baixíssimo custo.

O registro da temperatura é feito diretamente em uma planilha de dados Excel que vai recebendo valores de tempo e temperatura, em tempo real (SOKOLOFF; LAWS; THORNTON, 2007), coletados através de uma conexão tipo serial vinda de uma placa Arduino uno que faz a leitura do sinal de dois sensores tipo LM35 (taxa de uma leitura por segundo), um que monitora a temperatura do porta-amostra e outro que monitora a temperatura da blindagem. No caso da blindagem, o sensor foi posto em contato direto com a parede de alumínio do cilindro através da pasta térmica. $\mathrm{O}$ monitoramento ajuda a garantir a estabilidade pretendida para a temperatura da blindagem. Os códigos escritos para a leitura dos sinais elétricos dos sensores LM35 através da placa Arduino estão mostrados no anexo deste trabalho.

Os valores dispostos na planilha Excel podem ser observados através de gráficos de temperatura versus tempo dinamicamente o que ajuda a monitorar o andamento das medidas. A Fig. 5 apresenta a disposição dos constituintes do porta-amostra e o esquema da ligação elétrica do sensor de temperatura na placa Arduino Uno.

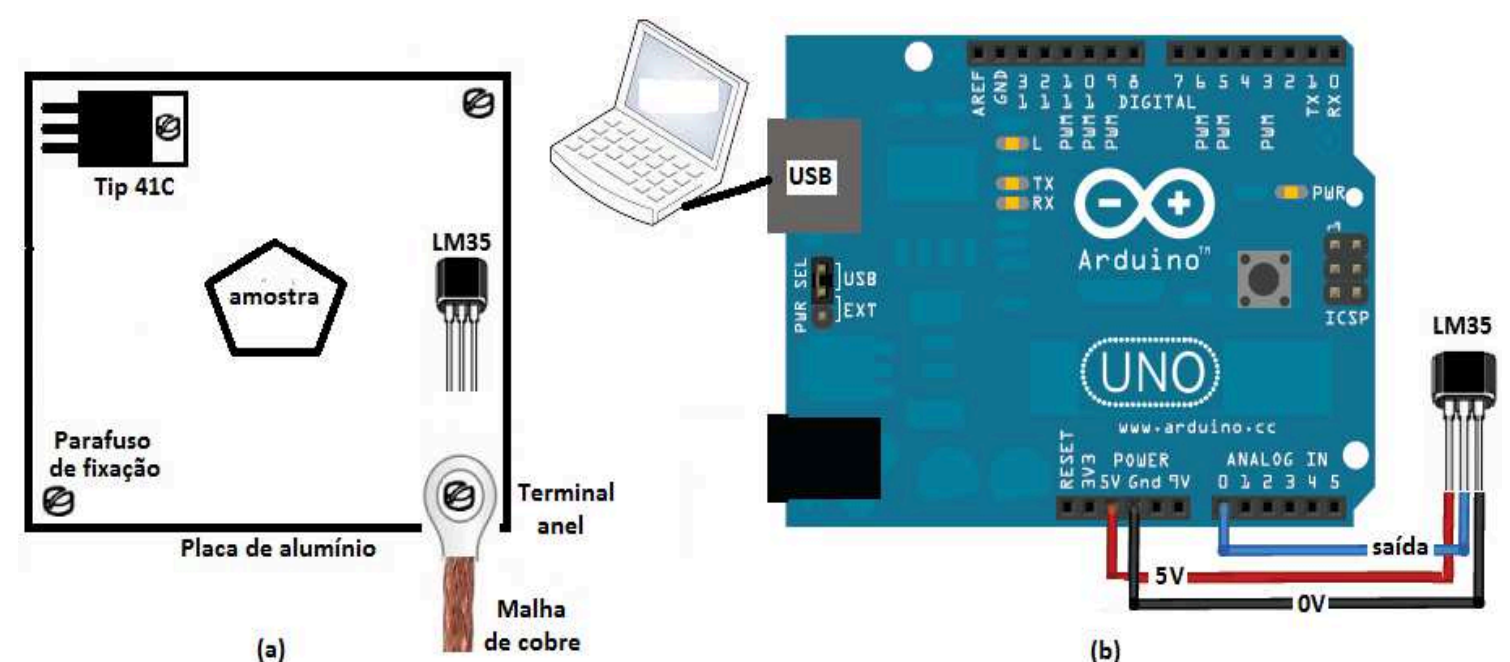

Fig. 5 - Em (a) a disposição dos constituintes do porta-amostra e em (b) a ligação simples de um sensor LM35 na placa Arduino Uno (SOUZA, 2011). O TIP 41C é fixado por baixo da lâmina de alumínio. 
Na Fig. 6, é possível ver uma foto do protótipo do calorímetro de relaxação. É possível notar que o porta-amostra é fixado por dois parafusos a uma placa de isopor de $2,0 \mathrm{~cm}$ de espessura colocada no fundo da caixa de isopor receptáculo do calorímetro. Também podemos ver que o cilindro metálico que serve de blindagem é colocado em um sulco feito na placa de isopor visando a sua estabilização durante a medida. Outra placa de isopor também medindo $2,0 \mathrm{~cm}$ de espessura tem um orifício em seu centro para a passagem da parte superior do cilindro metálico que fará contato térmico (via pasta térmica) com o reservatório que contém o gelo. As dimensões desta placa permitem um ajuste adequado para uma vedação parcial da parte superior da caixa de isopor com o meio externo.
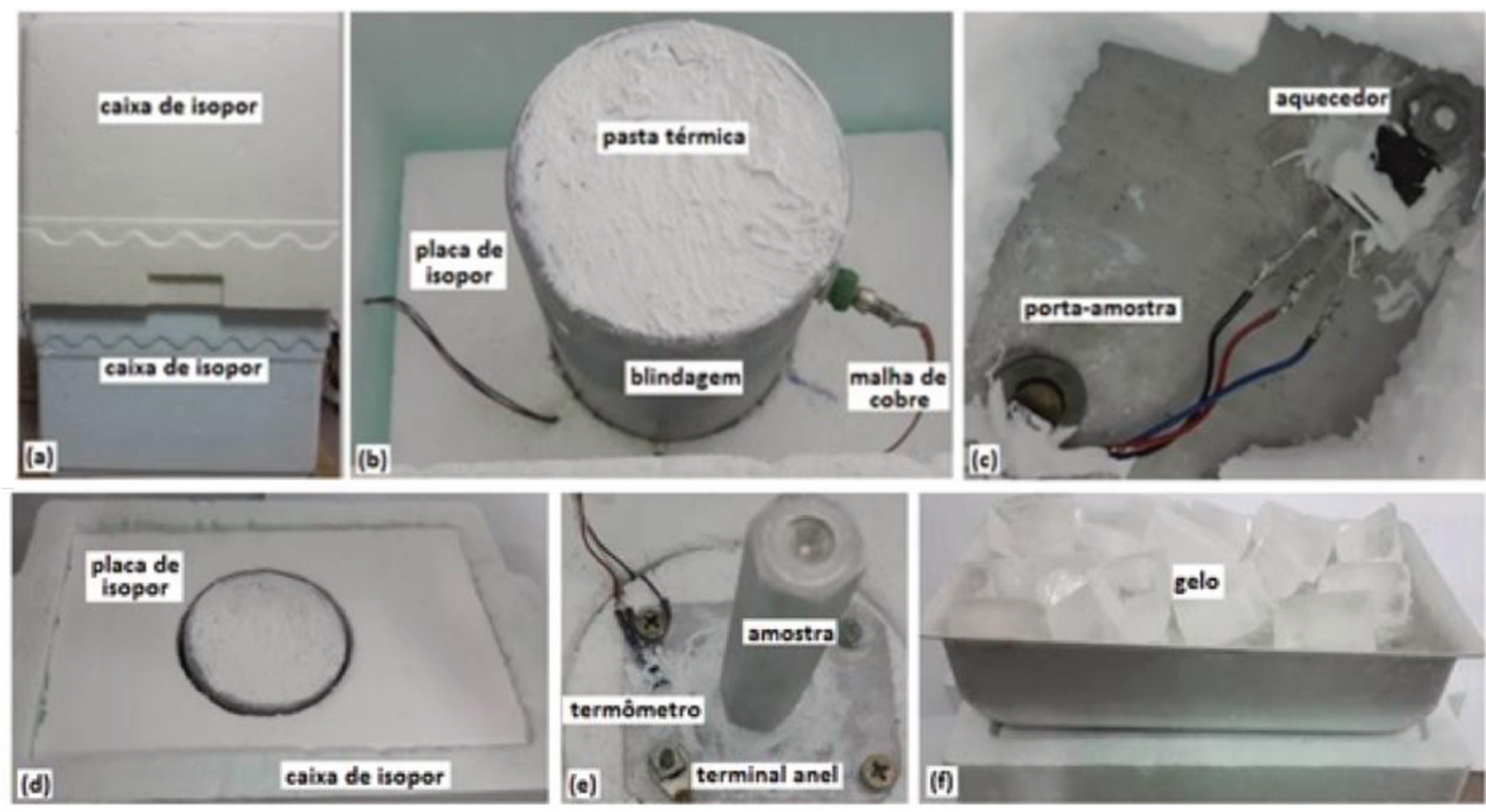

Fig. 6 - Fotos do protótipo do calorímetro de relaxação. Em (a) o receptáculo do porta-amostra, em (b) a blindagem térmica, em (c) a visão do aquecedor, em (d) a placa de isopor que fecha o calorimetro, em (e) a amostra e o termômetro acoplando e em (f) o reservatório de gelo (reservatório frio).

\section{Resultados e discussões}

O primeiro procedimento a ser realizado anteriormente às medidas de calor específico é a calibração do porta-amostra visando a determinação da potência do aquecedor e a subtração da capacidade térmica do porta-amostra da capacidade térmica do conjunto amostra mais portaamostra. Para isto, utilizamos uma amostra de alumínio comum de 43,2 g que servirá como padrão conhecido de capacidade térmica. A massa desta amostra padrão escolhida é cerca de 12 vezes maior que a massa do porta-amostra. Esta relação é desejável para que prepondere a maior contribuição da capacidade térmica da amostra em relação a capacidade térmica do porta- 
amostra. O valor de calor específico do alumínio utilizado nesta calibração foi de $896 \mathrm{~J} /(\mathrm{kg} . \mathrm{K})$ (DUNN, 2013). Todas as amostras são fixadas com pasta térmica ao porta-amostra.

A potência efetiva do aquecedor foi determinada através do uso da Equação 15 obtendo 3,52 W, trabalhando na faixa de temperaturas apontadas. A tabela 1 mostra os valores para a variação máxima de temperatura $\left({ }^{\circ} \mathrm{C}\right.$ ) e tempo de relaxação $\boldsymbol{\tau}$ (s) obtidos para apenas o porta-amostra e para o porta-amostra mais amostra de alumínio. A incerteza de medida para os valores de temperatura é de $0,25^{\circ} \mathrm{C}$. Foram feitas cinco medidas de calor específico buscando determinar a média a ser utilizada no cálculo da potência efetiva do aquecedor, veja a tabela 1.

Tabela 1 - Valores relativos às medidas de variação máxima de temperatura e tempo de relaxação para o porta-amostra e o porta-amostra contendo uma amostra de alumínio padrão. Os valores de desvio padrão da média apontam para a boa reprodutibilidade das medidas.

\begin{tabular}{|l|l|l|l|l|l|}
\hline Porta-amostra & $\begin{array}{c}\Delta \mathbf{T}_{\max } \\
\left({ }^{\mathbf{C}} \mathbf{C}\right)\end{array}$ & $\boldsymbol{\tau}(\mathbf{s})$ & $\begin{array}{c}\text { Porta-amostra }+ \\
\text { amostra de alumínio }\end{array}$ & $\begin{array}{c}\Delta \mathbf{T}_{\max } \\
\left({ }^{\mathbf{}} \mathbf{C}\right)\end{array}$ & \multicolumn{1}{c|}{$(\mathbf{s})$} \\
\hline $\mathbf{1}$ & 70,72 & $205,5 \pm 0,4$ & 1 & 55,52 & $753 \pm 2$ \\
\hline $\mathbf{2}$ & 70,91 & $204,5 \pm 0,4$ & 2 & 54,14 & $720 \pm 1$ \\
\hline $\mathbf{3}$ & 73,75 & $206,3 \pm 0,5$ & 3 & 52,91 & $801 \pm 1$ \\
\hline $\mathbf{4}$ & 72,95 & $207,2 \pm 0,4$ & 4 & 55.62 & $735 \pm 2$ \\
\hline $\mathbf{5}$ & 71,61 & $204,9 \pm 0,7$ & 5 & 54,53 & $753 \pm 2$ \\
\hline Média & 71,99 & 205,7 & Média & 54,54 & 752,4 \\
\hline $\begin{array}{l}\text { Desvio padrão } \\
\text { da média }\end{array}$ & 0,59 & 0,49 & Desvio padrão da & 0,50 & 13 \\
\hline
\end{tabular}

O desempenho do calorímetro foi avaliado através da medida do calor específico de três amostras metálicas, ferro $(\mathrm{m}=170,2 \mathrm{~g})$, chumbo $(\mathrm{m}=95,6 \mathrm{~g})$ e latão $(\mathrm{m}=250,0 \mathrm{~g})$. A tabela 2 apresenta os valores de variação máxima de temperatura e tempo de relaxação para as amostras de ferro, latão e chumbo. $\mathrm{O}$ valor do calor específico foi calculado utilizando as Equações 12,13 e 14 . 
Tabela 2 - Valores relativos às medidas de variação máxima de temperatura e tempo de relaxação para as amostras de ferro, latão e chumbo.

\begin{tabular}{|c|c|c|c|c|c|c|c|c|c|}
\hline $\begin{array}{c}\text { Corpo de } \\
\text { prova }\end{array}$ & $\begin{array}{c}\Delta \mathrm{T}_{\max } \\
\left({ }^{\circ} \mathrm{C}\right)\end{array}$ & $\begin{array}{c}\tau \\
(s)\end{array}$ & \begin{tabular}{c}
$\mathrm{C}_{\text {Ferro }}\left(\mathrm{Jkg}^{-1} \mathrm{~K}^{-1}\right)$ \\
\hline 1
\end{tabular} & $\begin{array}{c}\Delta \mathrm{T}_{\max } \\
\left({ }^{\circ} \mathrm{C}\right)\end{array}$ & $\begin{array}{c}\tau \\
(s)\end{array}$ & $\begin{array}{c}\mathrm{C}_{\text {latão }} \\
\left(\mathrm{J} \mathrm{kg-l} \mathrm{K}^{-1}\right)\end{array}$ & $\begin{array}{c}\Delta \mathrm{T}_{\max } \\
\left({ }^{\circ} \mathrm{C}\right)\end{array}$ & $\begin{array}{c}\tau \\
(s)\end{array}$ & $\begin{array}{c}\mathrm{C}_{\text {chumbo }} \\
\left(\mathrm{Jkg}^{-1} \mathrm{~K}^{-1}\right)\end{array}$ \\
\hline 2 & 54,57 & $1436 \pm 1$ & 494 & 50,56 & $1555 \pm 1$ & 389 & 63,44 & $413 \pm 1$ & 134 \\
\hline 3 & 55,25 & $1341 \pm 1$ & 452 & 52,04 & $1538 \pm 1$ & 377 & 63,00 & $431 \pm 1$ & 147 \\
\hline 4 & 56,28 & $1392 \pm 1$ & 477 & 51,93 & $1563 \pm 1$ & 385 & 64,81 & $429 \pm 1$ & 138 \\
\hline 5 & 54,50 & $1469 \pm 1$ & 490 & 52,26 & $1550 \pm 2$ & 377 & 64,2 & $404 \pm 1$ & 126 \\
\hline Média & 55,34 & 1404 & 473 & 51,37 & 1559 & 389 & 63,83 & 417 & 134 \\
\hline $\begin{array}{c}\text { Desvio } \\
\text { padrão } \\
\text { da média }\end{array}$ & $\approx 1,7$ & $\approx 4,6$ & $\approx 4,4$ & $\approx 2,5$ & $\approx 1,8$ & $\approx 4,3$ & $\approx 1,5$ & $\approx 3,3$ & $\approx 9,4$ \\
\hline
\end{tabular}

Utilizando o valor médio calculado de $71,99^{\circ} \mathrm{C}$ para a variação máxima de temperatura do porta-amostra e a potência do aquecedor que foi medida neste trabalho, podemos fazer uma estimativa da condutância térmica efetiva total do sistema (k) pela Equação 10, obtendo 4,89 x $10^{-2} \mathrm{~W} /{ }^{\circ} \mathrm{C}$. Este resultado está associado principalmente a malha de cobre que faz a transferência de calor do porta-amostra para a blindagem e também ao ar que envolve o porta-amostra e blindagem e em menor escala através da radiação térmica.

Na tabela 3 podemos ver os desvios dos valores médios de calor específicos medidos e os valores encontrados na literatura.

Tabela 3 - Desvios percentuais entre os valores médios de calor específicos medidos neste trabalho e valores encontrados na literatura (CRC, 2017) para temperatura ambiente. Dados de calor específicos em unidades de $\mathrm{J} /(\mathrm{kg} \mathrm{K})$.

\begin{tabular}{|c|c|c|c|}
\hline & Ferro & Latão & Chumbo \\
\hline Este trabalho & 473 & 389 & 134 \\
\hline Literatura & 449 & 385 & 130 \\
\hline Desvio (\%) & 5,3 & 1,1 & 3,1 \\
\hline
\end{tabular}

Da forma como foi concebido o projeto, o sistema termodinâmico que se pode montar é sempre único, apresentando características próprias de funcionamento e desempenho. $\mathrm{O}$ fato de que a experiência decorre sempre em condições de atmosfera não completamente controlada em aspectos como pressão (sistema não evacuado), temperatura (temperatura ambiente entre $17^{\circ} \mathrm{C}$ e $18^{\circ} \mathrm{C}$ durante os testes), umidade (umidade relativa do ar), etc., espera-se que ocorram pequenas diferenças nos resultados de variação máxima de temperatura e de tempo de relaxação 
do sistema. Contudo a reprodutibilidade das medidas pode ser considerada satisfatória observando-se os valores de desvio máximo percentual entre os valores medidos e a média calculada (ver tabela 2).

As amostras de ferro, latão e alumínio usadas neste trabalho foram obtidas em sucata de uma tornearia mecânica. A amostra de chumbo é do tipo "chumbada" de pescaria. Todos os metais são comerciais, não existindo nenhuma garantia de alto grau de pureza dos corpos de prova. Este fato também influência nos valores dos desvios encontrados para o calor específico medido frente a literatura (ver tabela 3). Podemos comparar o grau de desvio dos resultados encontrados neste trabalho com relação ao valor da literatura comparando com outras propostas de medidas encontradas na literatura (SILVA, 2003). A Fig. 7 apresenta gráficos das medidas de variação de temperatura em função do tempo nos regimes de aquecimento e resfriamento para as amostras utilizadas neste trabalho. Os valores de variação máxima de temperatura, $\Delta T_{\max }$ $\left({ }^{\circ} \mathrm{C}\right)$, são obtidos subtraindo a temperatura máxima atingida no patamar do regime de aquecimento da temperatura do patamar de temperatura de partida quando o sistema estabiliza em baixa temperatura, antes da aplicação do pulso térmico. A temperatura máxima e a temperatura mínima são obtidas através de um ajuste numérico linear nas faixas mencionadas. O tempo de relaxação, $\tau$ (s), é obtido através do ajuste numérico da equação 9 aos pontos experimentais no regime de resfriamento, ou seja, após a fonte de calor cessar sua emissão e a curva de temperatura versus tempo alcançar o patamar máximo. O ajuste mencionado deverá se dar até a curva alcançar o patamar de temperatura mínima, o que significa estar novamente em equilíbrio térmico com a blindagem.

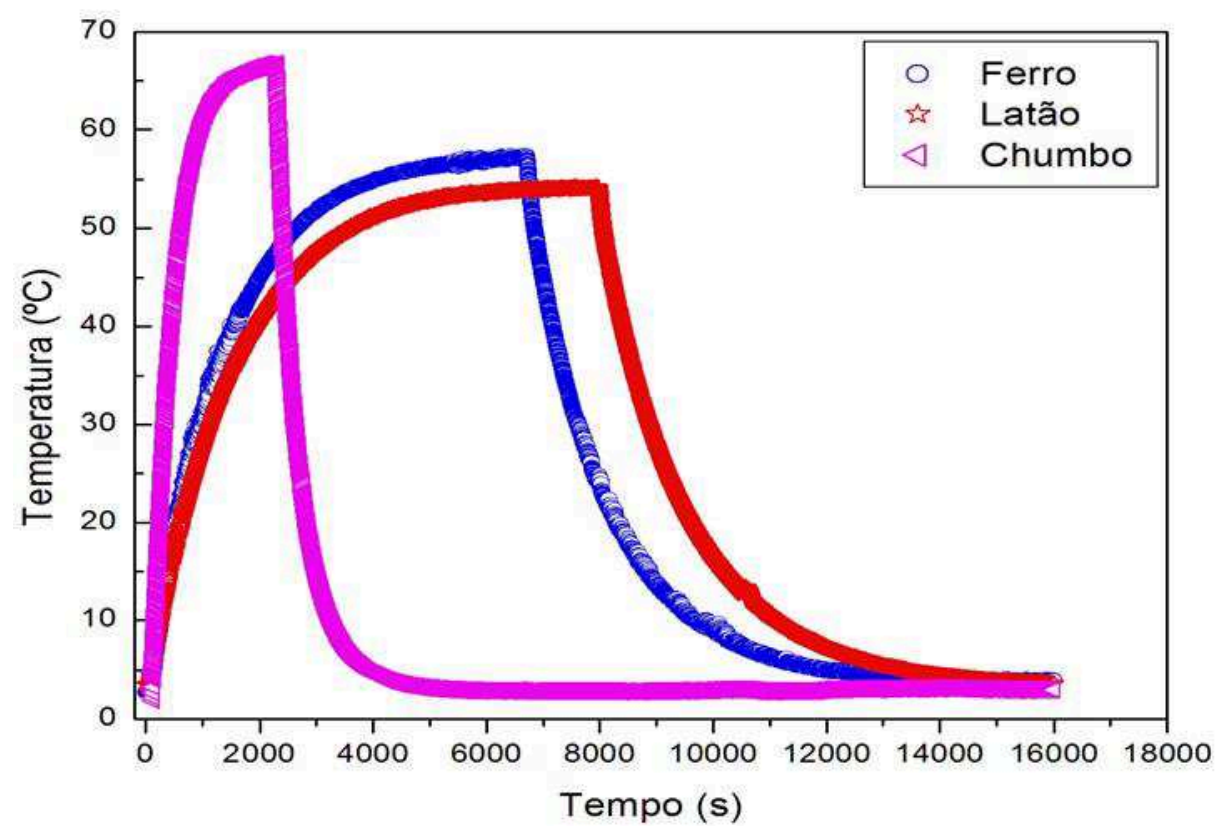

Fig. 7 - Gráficos de temperatura versus tempo nos regimes de aquecimento e resfriamento obtidos para as amostras de ferro, latão e chumbo. 
A Fig. 8 apresenta as curvas de temperatura versus tempo (e ajustes numéricos) no regime de resfriamento obtidos para as amostras de ferro, latão e chumbo.

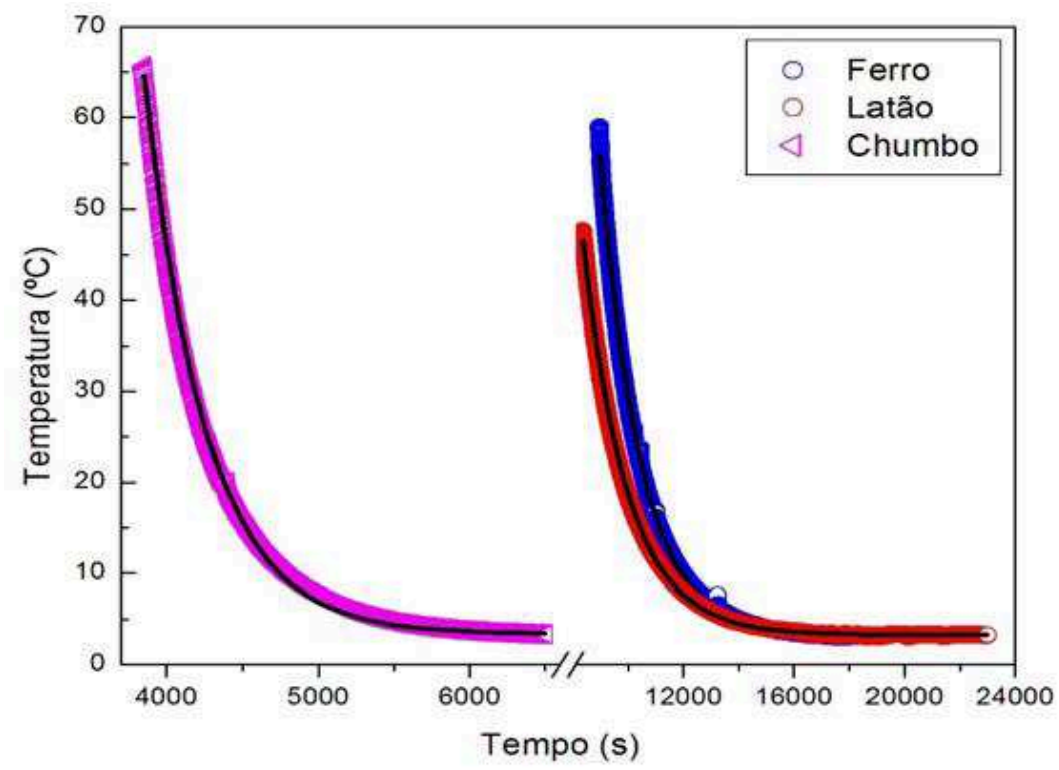

Fig. 8-Gráficos de temperatura versus tempo no regime de resfriamento obtidos para as amostras de ferro, latão e chumbo.

\section{Considerações finais}

Apresentamos as características de constituição física e funcionamento de um calorímetro de relaxação adequado para equipar um laboratório didático de ensino de física experimental. O projeto pode ser desenvolvido mesmo em laboratórios modestos de ensino de Física tanto com a finalidade de inovar e incrementar práticas experimentais como quanto para incentivar estudantes a trabalhar em projetos interdisciplinares onde o aluno pode desenvolver conhecimentos sobre sensores eletrônicos, microcontroladores e sua programação computacional e os limites de validade da informação numérica experimental. Claro que o projeto sempre deve estar adequado ao nível de escolaridade pertinente, seja ela na educação básica, técnica ou superior.

\section{Referências}

ANDERSON, H. A.; URBINA, U. L. Constructing a Relaxation Calorimeter. In: THE NATIONAL CONFERENCE ON UNDERGRADUATE RESEARCH, 2013, La Crosse. Proceedings... Asheville: University of North Carolina, 2013. p. 488-496.

AXT, R.; GUIMARÃES, V. H. Física experimental: Manual de laboratório para Mecânica

e Calor. 2. Ed. Porto Alegre: Editora da Universidade, 1991. 92 p. 
BORGES, T. A. Novos rumos para o laboratório escolar de ciências. Caderno Brasileiro de Ensino de Física, Florianópolis, v. 19, n. 3, p. 291-313, dez. 2002.

DUNN, W. C. Fundamentos de Instrumentação Industrial e Controle de Processos. Porto Alegre: Bookman, 2013. 344 p.

CRC. Handbook of Chemistry and Physics, W. M. Haynes ed. CRC Press, Boca Raton, FL, 2017.

SILVA, L. F.; FIGUEIRA, J. S.; VEIT, E. A. Atividades de aquisição automática de dados no laboratório de física da escola do ensino médio. In: SIMPÓSIO NACIONAL EM ENSINO DE FÍSICA, XVI, 2005, Rio de Janeiro. Anais... São Paulo, SBF, CD.

SÉRÉ, M. G.; COELHO, S. M.; NUNES, A. D. O papel da experimentação no Ensino de Física. Caderno Brasileiro de Ensino de Física, Florianópolis, v. 20, n. 1, p. 30-42, abril. 2003.

SILVA, W. P.; PRECKER J. W.; SILVA, C. M. D. P. S.; SILVA, D. D. P. S.; SILVA, C. D. P. S. Medida de calor especifico e Lei de Resfriamento de Newton: Um refinamento na análise dos dados experimentais, Revista Brasileira de Ensino de Física, São Paulo, v. 25, n. 4, p. 392-398, dez. 2003.

SOKOLOFF, D. R.; LAWS, P. W. THORNTON, R. K. Real Time Physics: active learning labs transforming the introductory laboratory. European Journal of Physics, v. 28, n. 3, p. S83S94, abr. 2007.

SOUZA, A. R. et al. A placa Arduino: uma opção de baixo custo para experiências de física assistidas pelo PC. Revista Brasileira de Ensino de Física, São Paulo, v. 33, n. 1, p. 1702-011702-05, jan/março 2011.

STEIMACHER, A. Desenvolvimento e caracterização de vidros aluminosilicato de cálcio dopados com Nd ${ }^{3+}$. 2008. 135 f. Tese (Doutorado em Física) - Instituto de Física, Universidade Estadual de Maringá, Maringá.

YOUNG, H. D.; FREEDMAN, R. A. Temperatura e Calor. In: Física II: Termodinâmica e ondas. 10. ed. São Paulo: Pearson Addison Wesley, 2003. cap. 15, p. 103-141.

\section{Anexo}

Apresentamos o código computacional que foi desenvolvido visando a leitura do tempo e dos níveis de tensão elétrica dos sensores LM35 através da placa Arduino, o processamento desta informação e sua transmissão via porta serial do computador. O sinal de tensão elétrica analógico variável que é colhido dos sensores dinamicamente é convertido em sinal 
digital pelo conversor de 10 bits presente nas portas A0 e A1. Após esta conversão, a programação realiza uma operação matemática que converte o número binário em unidades de ${ }^{\circ} \mathrm{C}$ antes de seguir para o computador via porta serial.

O programa computacional PLX-DAQ desenvolvido e disponibilizado gratuitamente pelo site da empresa PARALLAX INC. constitui uma ferramenta útil para viabilizar a leitura dos dados enviados pela placa Arduino. O PLX-DAQ pode ler até 26 canais de dados simultaneamente, em tempo real, através de uma taxa de até $128 \mathrm{kbps}$. Os números que chegam do Arduino são dispostos numa tabela do programa Excel, disponibilizando assim o gráfico atualizável da grandeza física que se esteja medindo (em nosso caso o tempo e a temperatura). Além da programação revelada neste anexo, que viabiliza a comunicação do Arduino com o PLXDAQ, o site da empresa PARALLAX. INC, em:<www.parallax.com/tabid/393/Default.aspx>, disponibiliza outros exemplos que ilustram a potencialidade desta ferramenta. Na Fig. 9 mostramos a caixa de controle principal do PLX-DAQ.

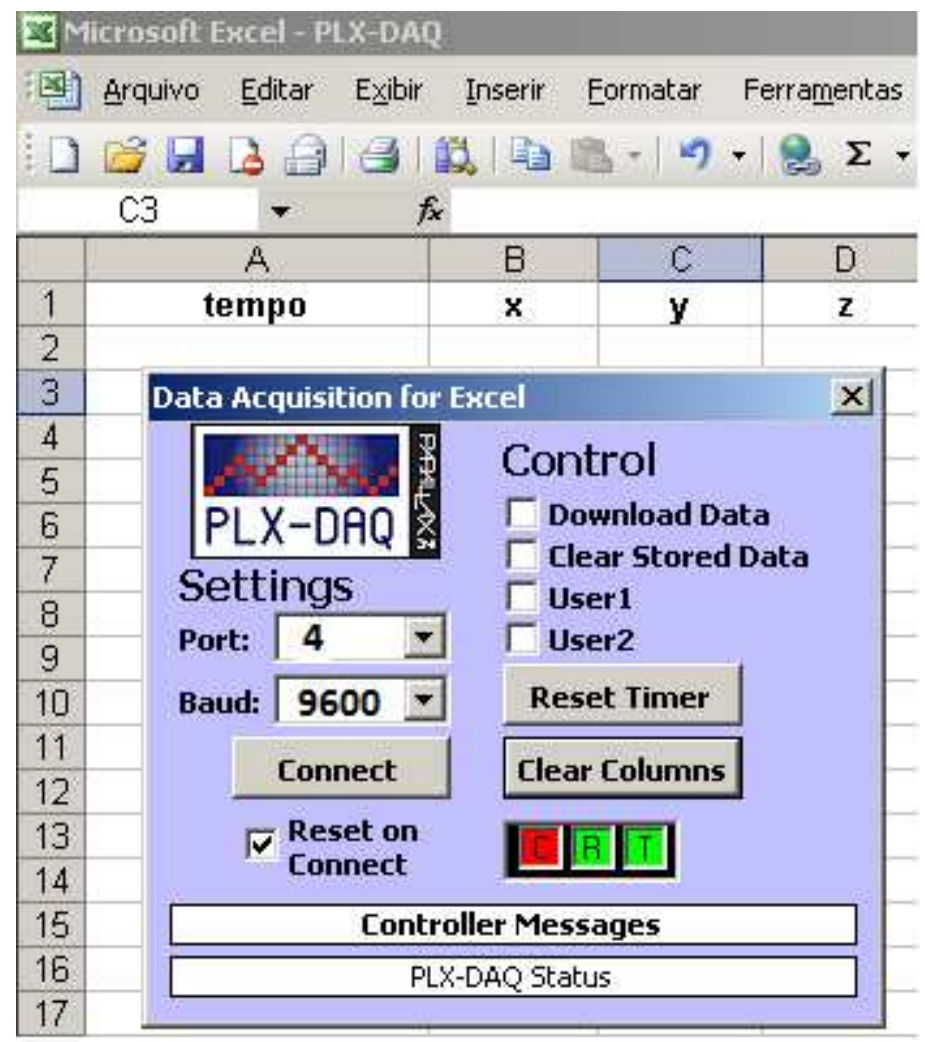

Fig. 9 - Caixa de controle inicial do programa PLX-DAQ. Importante definir a taxa "Baund" e a porta serial por onde será transmitida a informação.

No código apresentado na Fig. 10, primeiro vemos a definição das variáveis importantes de tempo, temperaturas de porta-amostra e blindagem e de tensão elétrica de leitura de saída dos termômetros LM35. Em seguida temos a definição da taxa de transferência de dados "Serial.begin (9600)" que deve ser a mesma a ser escolhida na caixa de controle do PLX-DAQ, 
veja a Fig. 9. Este valor pode ser aumentado até 128000 significando aumento da capacidade de dados transferidos por segundo. Ainda no "void setup" encontramos o início da formatação dos dados que são enviados pela serial e devidamente reconhecidos pelo PLX-DAQ. Esta formatação é específica e não pode ser alterada em sua estrutura para que os dados enviados sejam corretamente interpretados pelo PLX-DAQ. No "void loop" vemos a definição do inteiro "i" que contará 1000 leituras das temperaturas "temp" e "tempb". Estes valores comporão uma média aritmética simples minimizando assim a presença de dados espúrios oriundos de ruído nas medidas. O código prossegue anotando a base de tempo do microcontrolador, lida na função "millis" que fornece o tempo em milissegundos. Os dados medidos seguem para o Excel pelo comando "serial.println" veja a Fig. 11. No exemplo que apresentamos, o "loop" se dá incrementando um contador de unidade em unidade até o limite de, por exemplo, 1000000. Esse valor representará o número máximo de linhas que serão populadas com os dados medidos. Uma vez alcançado este limite, a planilha volta para a primeira linha apagando os dados remanescentes e atualizando a informação. Segue assim indefinidamente.

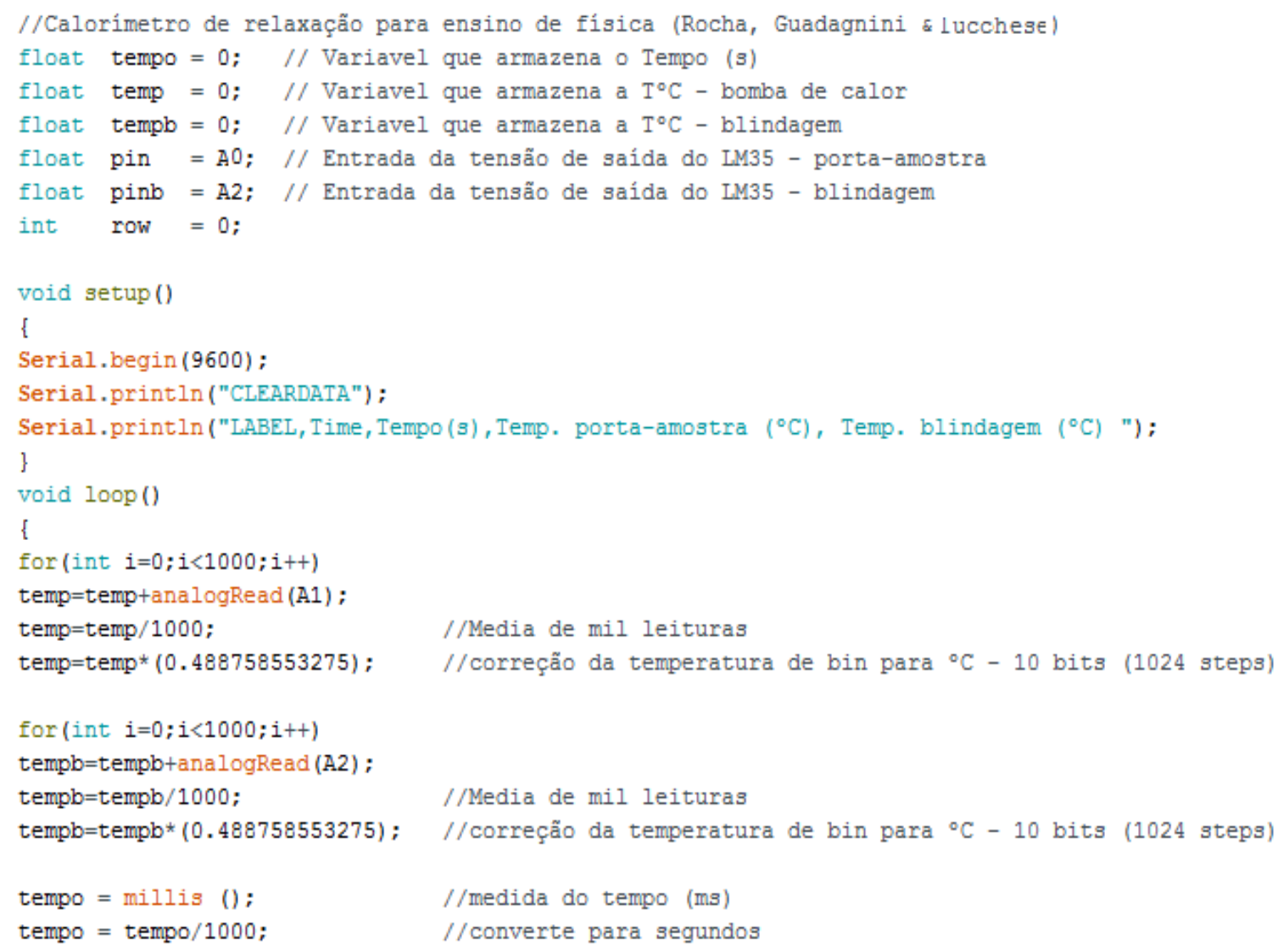

Fig. 10 - Primeira etapa do código computacional utilizado no microcotrolador da placa Arduino. 


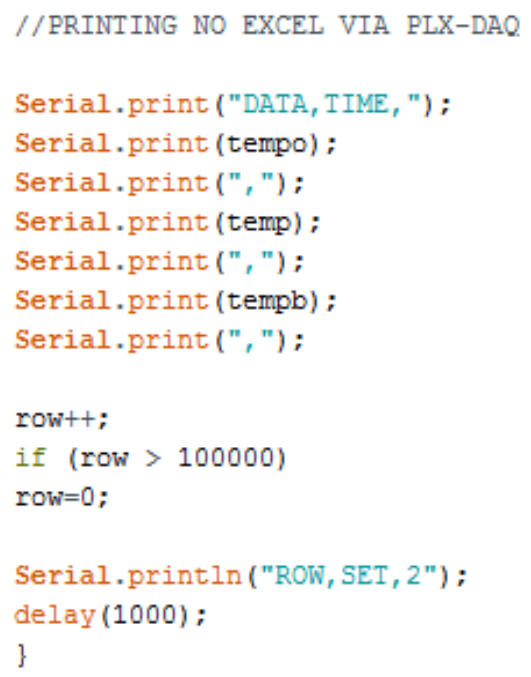

Fig. 11 - Segunda etapa do código computacional utilizado no microcontrolador da placa Arduino. 